\title{
Experimental search for solar hidden photons in the eV energy range using kinetic mixing with photons
}

\author{
T. Mizumoto ${ }^{a}$ R. Ohta ${ }^{b}$ T. Horie ${ }^{b}$ J. Suzuki ${ }^{b}$ Y. Inoue ${ }^{c}$ \\ M. Minowa ${ }^{b, d}$
}

${ }^{a}$ Department of Physics, Graduate School of Science, Kyoto University, Kitashirakawa-Oiwakecho, Sakyo-ku, Kyoto 606-8502, Japan

${ }^{b}$ Department of Physics, School of Science, The University of Tokyo, 7-3-1 Hongo, Bunkyo-ku, Tokyo 113-0033, Japan

${ }^{c}$ International Center for Elementary Particle Physics, The University of Tokyo, 7-3-1 Hongo, Bunkyo-ku, Tokyo 113-0033, Japan

${ }^{d}$ Research Center for the Early Universe (RESCEU), School of Science, The University of Tokyo,

7-3-1 Hongo, Bunkyo-ku, Tokyo 113-0033, Japan

E-mail: minowa@phys.s.u-tokyo.ac.jp

\begin{abstract}
We have searched for solar hidden photons in the eV energy range using a dedicated hidden photon detector. The detector consisted of a parabolic mirror with a diameter of $500 \mathrm{~mm}$ and a focal length of $1007 \mathrm{~mm}$ installed in a vacuum chamber, and a photomultiplier tube at its focal point. The detector was attached to the Tokyo axion helioscope, Sumico which has a mechanism to track the sun. From the result of the measurement, we found no evidence for the existence of hidden photons and set a limit on the photon-hidden photon mixing parameter $\chi$ depending on the hidden photon mass $m_{\gamma^{\prime}}$.
\end{abstract}

Keywords: hidden, photon, solar 


\section{Contents}

1 Introduction $\quad 1$

2 Experimental apparatus $\quad 3$

3 Measurement and analysis $\quad 4$

4 Conclusion $\quad 10$

\section{Introduction}

A hidden photon is the gauge boson of a hypothetical hidden local U(1) symmetry. Such symmetries arise in a generic prediction of many extensions of the standard model, especially in those based on string theory[1]. If the hidden photons are massive but the mass is small enough, the hidden photons have rich phenomenology[2] at low energy scales.

The dynamics of the photon-hidden photon system with kinetic mixing is described by the following Lagrangian,

$$
\mathcal{L}=-\frac{1}{2} \chi F_{\mu \nu} B^{\mu \nu}
$$

where $F_{\mu \nu}$ and $B_{\mu \nu}$ represent the ordinary and the hidden photon field, respectively[24]. When the hidden photon has non-zero mass $m_{\gamma^{\prime}}$, it leads to photon-hidden photon oscillations similar to vacuum neutrino ocillations. In vacuum, hidden photon $\rightarrow$ photon transition probability $P_{\gamma^{\prime} \rightarrow \gamma}(\omega)$ is given by:

$$
P_{\gamma^{\prime} \rightarrow \gamma}(\omega)=4 \chi^{2} \sin ^{2}\left(\frac{\Delta q \ell}{2}\right)
$$

where $\omega$ is the energy of the photon, $\ell$ is the traveling path length and $\Delta q$ is the momentum transfer between the photon and hidden photon which is given by:

$$
\Delta q=\omega-\sqrt{\omega^{2}-m_{\gamma^{\prime}}^{2}} \sim \frac{m_{\gamma^{\prime}}^{2}}{2 \omega}
$$

assuming $m_{\gamma^{\prime}} \ll \omega$.

The matter effects modify the photon-hidden photon transition probability. Neglecting photon absorption, it can be written with the effective photon mass $m_{\gamma}[7,8]$ as

$$
P_{\gamma^{\prime} \rightarrow \gamma}(\omega)=\frac{4 \chi^{2} m_{\gamma^{\prime}}^{4}}{\left(m_{\gamma^{\prime}}^{2}-m_{\gamma}^{2}\right)^{2}+4 \chi^{2} m_{\gamma^{\prime}}^{4}} \times \sin ^{2}\left(\ell \times \frac{\sqrt{\left(m_{\gamma^{\prime}}^{2}-m_{\gamma}^{2}\right)^{2}+4 \chi^{2} m_{\gamma^{\prime}}^{4}}}{4 \omega}\right) .
$$


The effective photon mass $m_{\gamma}$ is defined by the following relation with the momentum $k$ of the photon and the refractive index $n$,

$$
m_{\gamma}^{2}=\omega^{2}-k^{2}=-\omega^{2}\left(\frac{k^{2}}{\omega^{2}}-1\right)=-\omega^{2}\left(n^{2}-1\right) .
$$

The probability gets lower as the matter density becomes higher due to the denominator of the oscillation amplitude.

Constraints on the massive hidden photon have been obtained from precision measurements of Coulomb's law $[2,5,6]$, from stellar cooling considerations [7, 8, 16], and from the photon regeneration or LSW(Light Shining through Walls) experiments. Recently, constraints on $\chi$ for the mass region $10^{-4} \mathrm{eV}<m_{\gamma^{\prime}}<10^{-2} \mathrm{eV}$ have been obtained from the results of the ALPS collaboration[9], the BMV collaboration[10], the GammeV collaboration[11], and the LIPSS collaboration[12]. More recently, a high energy solar hidden photon search with HP Ge detector[13] was reported. Solar axion search experiments are sensitive to the keV part of the solar spectrum of hidden photons and the latest CAST results[14, 15] have been translated into limits on the photon-hidden photon mixing parameter[16]. Bounds on models with additional new particles and a hidden photon at a low energy scale could be obtained from astrophysical considerations[17-19]. If hidden photons exist, production of them leads to distortions in the cosmic microwave background(CMB) spectrum. The CMB spectrum data provided by the Far Infrared Absolute Spectrophotometer (FIRAS) on board of the COBE constrained the hidden photon existence[20].

Since hidden photons can be produced through mixing with ordinary photons, the sun could be a source of low energy hidden photons. The coherence length of the photon-hidden photon oscillations is much shorter than the distance from the sun to the earth. Therefore, the transition probability is $2 \chi^{2}$ and the flux of hidden photons from the solar surface[16] is calculated to be

$$
\frac{\mathrm{d} \Phi^{s}}{\mathrm{~d} \omega} \simeq \chi^{2}\left(4.2 \times 10^{18}\right) \frac{\omega^{2}}{e^{\omega / T_{0}}-1} \frac{1}{\mathrm{eV}^{3} \mathrm{~cm}^{2} \mathrm{~s}} .
$$

In addition to the above flux, much higher flux of hidden photons is expected from photon-hidden photon oscillations in the bulk solar interior with a higher emitting volume and a higher temperature. For $m_{\gamma^{\prime}}$ well below the eV, one can use the following conservative estimate for the bulk component of the hidden photon flux at the earth[16]:

$$
\frac{\mathrm{d} \Phi^{b}}{\mathrm{~d} \omega} \simeq \chi^{2}\left(\frac{m_{\gamma^{\prime}}}{\mathrm{eV}}\right)^{4} 10^{32} \frac{1}{\mathrm{eVcm} \mathrm{cm}^{2} \mathrm{~S}} \quad \text { for } \quad \omega=1-5 \mathrm{eV}
$$

which exceeds the surface contribution except for masses $m_{\gamma^{\prime}} \leq 10^{-4} \mathrm{eV}$.

Recently, more refined estimation of the bulk flux is given by the same author[21, 22 ] taking into account the resonant production of hidden photons in a thin spherical solar shell, where the effective photon mass $m_{\gamma}$ is equal to the hidden photon mass $m_{\gamma^{\prime}}$. They claim that the resonant production dominates over the emission from the 


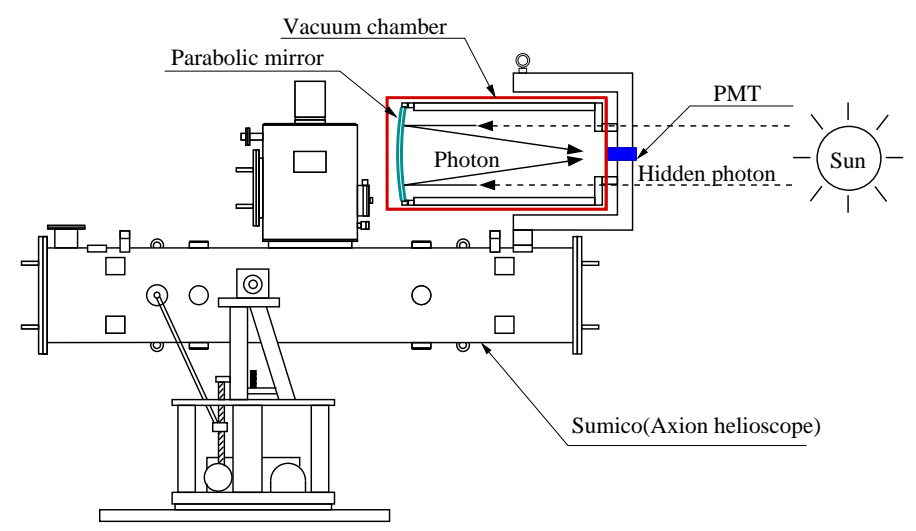

Figure 1. The schematic view of Sumico (Tokyo Axion Helioscope) and the solar hidden photon detector.

rest of the sun and gave hidden photon flux estimation for four typical cases of the hidden photon mass $m_{\gamma^{\prime}}=0,0.01,0.1$ and $1 \mathrm{eV}$.

In this paper, we report on the direct experimental search for the flux of solar hidden photons.

\section{Experimental apparatus}

For this experiment, we built a dedicated solar hidden photon detector and mounted it on Sumico, the Tokyo axion helioscope[25-28] as shown in fig. 1. Sumico has an altazimuth mount with a driving range from $-28^{\circ}$ to $28^{\circ}$ in altitudinal direction and $360^{\circ}$ in azimuth. Sumico is designed to search for the solar axion which is a particle introduced to solve the strong CP problem[23, 24].

The overall tracking accuracy is better than $0.5 \mathrm{mrad}$ both in altitudinal and azimuthal direction. Main components of the errors are a fluctuation of the turntable and a possible misalignment of the magnet aperture and the helioscope axis. The guidance of the helioscope movement is provided by the tracking software. In order to calculate the position of the sun, the U. S. Naval Observatory Vector Astronomy Subroutines (NOVAS-C ver 2.0.1)[29] is used. NOVAS-C calculates the topocentric position of the sun with less than 2 arcseconds $(9.7 \mu \mathrm{rad})$ error. The altitudinal origin was determined from a spirit level. While the sun is not directly visible from the laboratory in the basement floor, the azimuthal origin was first determined by a gyrocompass, which detects the north direction by the rotation of the earth within an error of $8 \operatorname{arcseconds}(39 \mu \mathrm{rad})$, and then it was introduced to the laboratory with a theodolite. The overall tracking error is negligible in our measurements.

The solar hidden photon detector consists of a vacuum chamber, a parabolic mirror and a single photon detector.

The vacuum chamber holds the conversion region in vacuum to keep the hidden photon $\rightarrow$ photon conversion probability high enough. It is a cylinder made of $1.5-\mathrm{mm}$ thick stainless steel plates with wrinkles on its side for the mechanical reinforcement. The inner diameter of the cylindrical vacuum chamber is $567 \mathrm{~mm}$ and its length is 
$1200 \mathrm{~mm}$. The cylinder can be divided into two parts: the cylinder main body with a closed bottom on one end and its lid on the other end. The lid is equipped with a vacuum gauge and an electronic thermometer. An ISO KF40 port is placed at the center of the lid and stands up toward the inside. The port is used to install a photomultiplier tube (PMT). A quartz glass vacuum window is attached to the port to transmit conversion photons to the PMT which is set at the atmospheric pressure side.

A parabolic mirror was used to collect the conversion photons to the PMT at its focal point. The mirror is made of soda glass and aluminium is deposited on the surface of the mirror. The mirror is $500 \mathrm{~mm}$ in diameter, $19 \mathrm{~mm}$ thick, $1007 \mathrm{~mm}$ focal length and the focal spot diameter is $1.5 \mathrm{~mm}$. The mirror is mounted on a mirror holder made of two aluminium rings. One holds the mirror directly by four clamps with silicon rubber pads. Another part of the mirror holder is attached to four channel steels which hold the parabolic mirror and its holder. Both are fixed to each other with three pairs of pushing and pulling bolts at the corners of an equilateral triangle, with which we can adjust the mirror axis so that the center of the PMT is on the optical axis. The reflectance of the parabolic mirror is measured by the manufacturer as a function of the wavelength of the photon. It is higher than $80 \%$ over the range between 300 and $650 \mathrm{~nm}$ with the maximum at around $400 \mathrm{~nm}$.

We used a photon counting PMT as a detector of the photon which is generated in the process of hidden photon $\rightarrow$ photon conversion. We selected a PMT, Hamamatsu Photonics R3550P because of its low dark count rate. It is a head-on type PMT and the tube size is $25 \mathrm{~mm}$ in diameter. It has a low noise bialkali photocathode whose effective area is $22 \mathrm{~mm}$ in diameter and it is sensitive to photons of wavelength range $300-650 \mathrm{~nm}$ with a peak quantum efficiency of $17 \%$.

Single and multi photon events detected by the PMT make current pulses which enter a charge-sensitive preamplifier (ORTEC 113) and a shaping amplifier (ORTEC 572). The signal is then sent to an ADC (Laboratory Equipment Corp. 2201A) and the multichannel analyser (MCA) spectrum is taken and recorded by a PC every $100 \mathrm{~s}$ live time.

The inner pressure of the vacuum chamber was measured by a vacuum gauge (Balzers PKR250). The temperatures of the PMT and the vacuum chamber were measured by Pt100 thermometers and recorded by another PC. During the solar tracking and background measurements, the inner pressure of the vacuum chamber was lower than $(5 \pm 2) \times 10^{-3} \mathrm{~Pa}$ at a temperature of $23^{\circ} \mathrm{C}$. The effect of this remaining gas on the conversion probability equation (1.4) is negligible.

\section{Measurement and analysis}

If a hidden photon is converted into a photon in the vacuum chamber, it would be detected by the PMT as a single photon event. Before starting the measurement, the shape of a single photon spectrum in the MCA was measured by illuminating the PMT with a blue LED with sufficiently low current pulses. It was fitted by a gaussian 


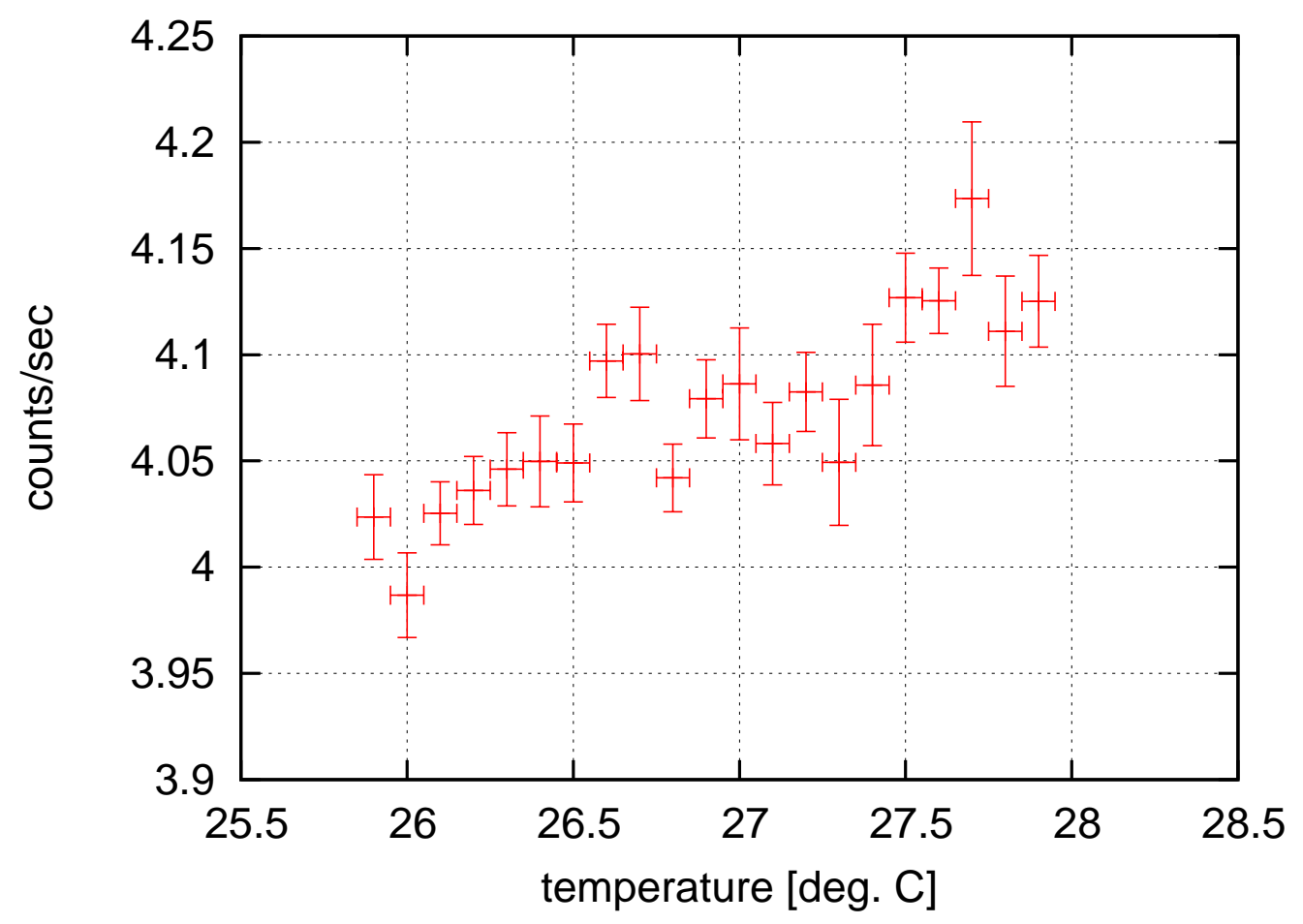

Figure 2. Temperature dependence of the dark count rate.

function and was later used as the template for the single photon analysis of the hidden photon search.

The solar tracking measurements were done around the time of sunrise and sunset with tracking time of about 5 hours each. Background measurements were also done before and after the solar tracking measurement by directing the detector away from the sun. All the measurements were done from October 26, 2010 till November 16, 2010 (22days).

To find out the possible evidence of solar hidden photons from the data of the measurement, we subtract the background spectrum from the solar tracking spectrum. We must eliminate some systematic effects which have nothing to do with the solar hidden photons.

It is well known that the dark count rate gets lower as time passes after an operating voltage is applied. We, therefore, waited for four days until the time dependence on the dark count rate became negligible.

Next, a temperature dependence of the dark count rate might cause a systematic effect on the background subtraction. Fig. 2 shows observed temperature dependence of the dark count rate. To avoid the effect, we subtracted background isothermally. First, we grouped the solar tracking- and background-spectra each with $100 \mathrm{~s}$ of live time into 21 temperature bins of $0.1^{\circ} \mathrm{C}$ interval each whose central values ranging from 25.9 to $27.9^{\circ} \mathrm{C}$. Then, we apply the background subtraction in every temperature bin and obtained 21 residual spectra. Fig. 3 shows the solar tracking data, background 

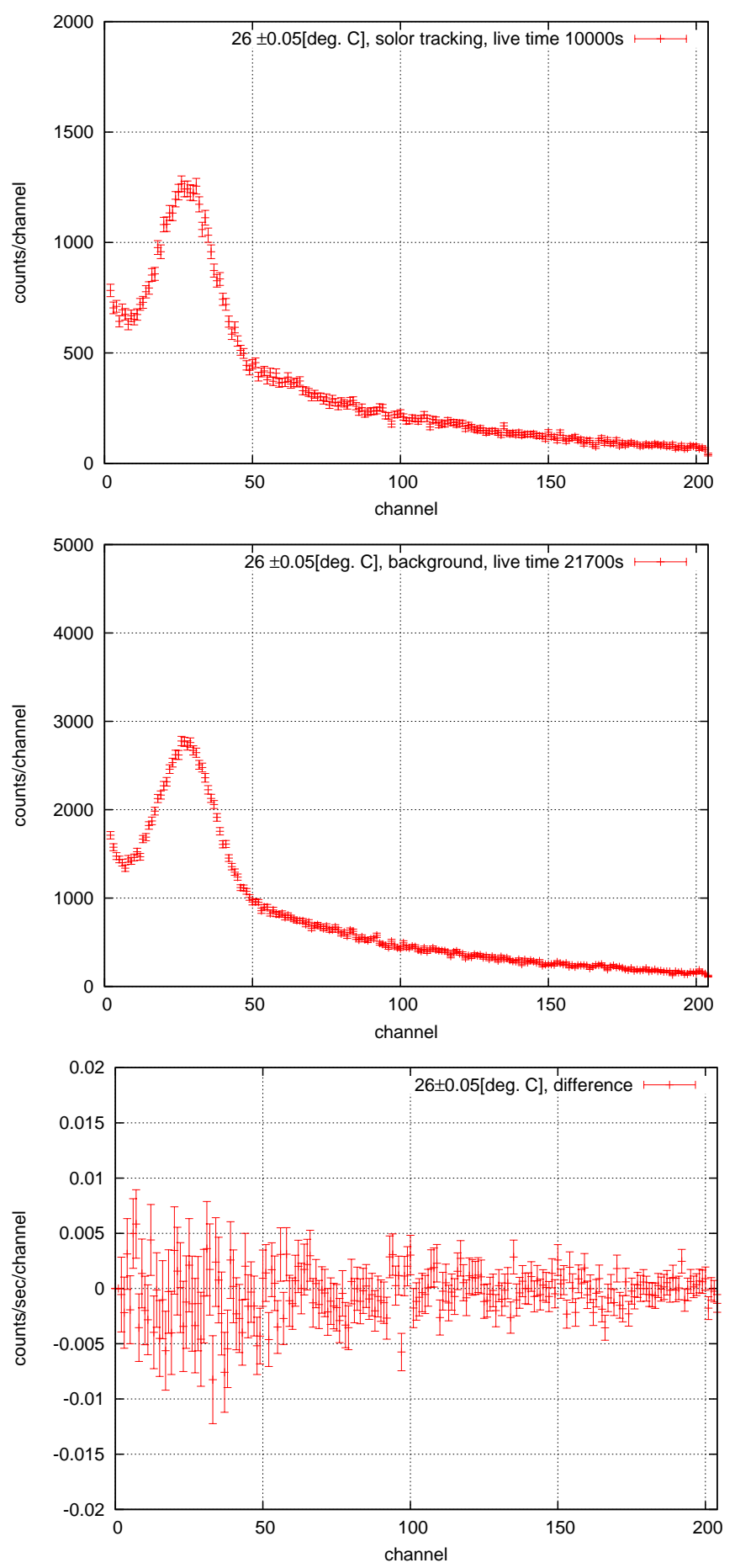

Figure 3. Spectrum examples (PMT temperature $26.0 \pm 0.05{ }^{\circ} \mathrm{C}$ ). Top: solar tracking spectrum. Middle: background spectrum. Bottom: spectrum after background subtraction.

data and residual spectrum in the temperature bin $26 \pm 0.05{ }^{\circ} \mathrm{C}$, as an example. The former two show peaks of single photoelectron events. Finally, we combined the residual 


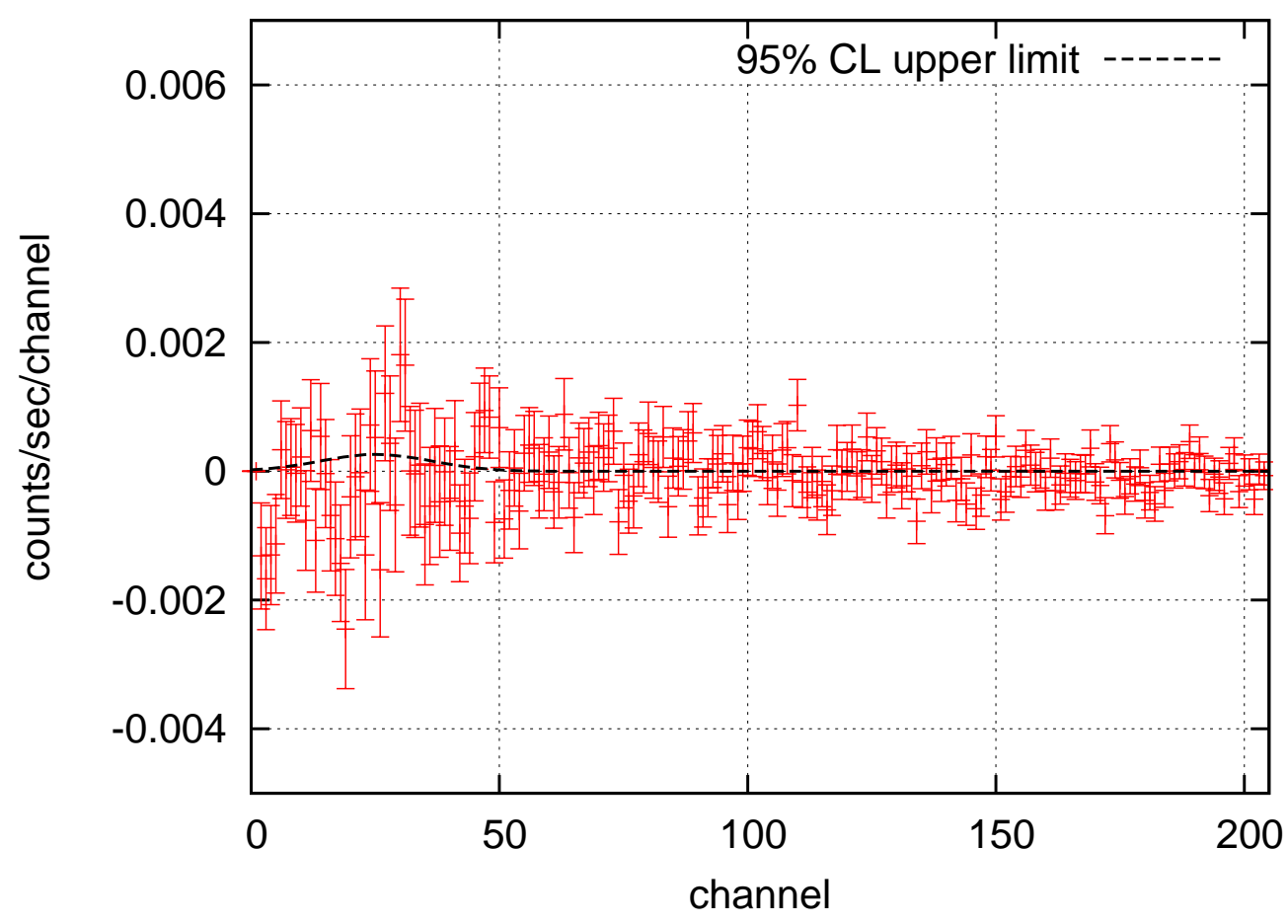

Figure 4. Total residual spectrum and the $95 \%$ confidence level upper limit.

spectra of all the temperature bins taking into account errors in them, and obtained the total residual spectrum.

In the above procedure, we took only the data during the holidays when the air conditioning system was switched off, because we observed abrupt room temperature changes on weekdays due to automatic switching of the air conditioning system of the building. The subtraction scheme might fail when temperature changes so quickly that the thermometer does not follow the PMT temperature.

The final result is shown in fig. 4 . We then estimated how many single photons could there be in the total residual spectrum by fitting the magnitude of the gaussian template function to it. The best fit was obtained with

$$
\left.\left.N_{\text {fit }}=(-7.9 \pm 6.5 \text { (stat. }) \pm 3.4 \text { (sys. }\right)\right) \times 10^{-3}\left[\mathrm{~s}^{-1}\right] .
$$

The systematic errors considered include an effect of Cherenkov light emitted in the quartz glass vacuum window and the PMT window by cosmic muons. They might come into the PMT and be observed as a single photon event. Since cosmic muons have directional dependence, background subtraction might fail to give a fake effect. The finite bin width of the temperature might cause a systematic effect as well. We would like to make the bin width as narrow as possible, but too narrow a binning is impractical. The systematic error is estimated with the adopted temperature bin width and the temperature dependence of the dark count rate estimated in fig. 2. The third thing to be considered is drift effect of the dark noise rate. As already described in 
Table 1. Systematic and statistical errors

\begin{tabular}{lr}
\hline item & value $($ counts $/ \mathrm{s})$ \\
\hline muonic Cherenkov light & $1.1 \times 10^{-3}$ \\
temperature bin & $2.8 \times 10^{-3}$ \\
dark noise drift & $1.5 \times 10^{-3}$ \\
statistical & $6.5 \times 10^{-3}$ \\
\hline total & $7.4 \times 10^{-3}$ \\
\hline
\end{tabular}

the above paragraph, we waited for four days to start the measurement until the dark count rate got stable. A residual drift of the dark count rate after four days could give a systematic error. Possible gain drift of the PMT was monitored by tracking the single photoelectron peak of the spectrum. It amounted to $\pm 2 \%$ during the measurement and could cause only negligible systematic error. All these systematic errors and the statistical error are estimated and summarized in table 1.

The 95\% confidence level upper limit to the hidden photon counting rate was estimated from the fitting taking the statistical and systematic errors into account;

$$
N_{\mathrm{UL} 95}=1.02 \times 10^{-2} \mathrm{~S}^{-1} .
$$

The obtained upper limit $N_{\mathrm{UL} 95}$ is now compared with the count rate $N_{\exp }$ expected by the hidden photon model with given parameters;

$$
\begin{aligned}
N_{\exp }=\int \mathrm{d} \omega \frac{\mathrm{d} \Phi}{\mathrm{d} \omega}\left(\chi, m_{\gamma^{\prime}}, \omega\right) & \times \eta_{\text {mirror }}(\omega) \eta_{\text {window }} \eta_{\mathrm{PMT}}(\omega) \\
& \times S P_{\gamma^{\prime} \rightarrow \gamma}\left(\chi, m_{\gamma^{\prime}}, \omega, n(p, T, \omega), \ell\right),
\end{aligned}
$$

where $\frac{\mathrm{d} \Phi}{\mathrm{d} \omega}$ is the solar hidden photon spectral flux at the surface of the earth, $\eta_{\text {mirror }}$ is the reflectance of the parabolic mirror, $\eta_{\text {window }}$ is the transmittance of the quartz glass window, $\eta_{\mathrm{PMT}}$ is the detection efficiency of the PMT, $S$ is the area of the conversion region of the experimental apparatus, $P_{\gamma^{\prime} \rightarrow \gamma}$ is the hidden photon to photon conversion probability in the apparatus and $n$ is the refractive index of the conversion region as a function of the pressure $p$ and the temperature $T$.

From equations (3.2) and (3.3), the upper limit to the mixing angle $\chi$ as a function of the hidden photon mass $m_{\gamma^{\prime}}$ is calculated. In the calculation, possible systematic errors in the parameters of equation (3.3) have been taken into account; $\eta_{\text {mirror }}, \eta_{\text {window }}$, $\eta_{\mathrm{PMT}}, S$ and $\ell$. They are relatively small compared to the systematic errors of $N_{\text {fit }}$.

For the solar hidden photon flux $\frac{\mathrm{d} \Phi}{\mathrm{d} \omega}$ in equation(3.3), we assumed two cases. One is the sum of the conservative estimations[16], equations(1.6) and (1.7). The other is the more refined flux calculation[21, 22] with the resonant hidden photon production in the spherical solar shell beneath the surface.

Thus obtained $95 \%$ confidence level upper limit to the mixing angle $\chi$ is shown in fig. 5. We also show the limits set by other experiments with filled areas. The regions excluded by precision measurements of Coulomb's law [5, 6], LSW(Light Shining 


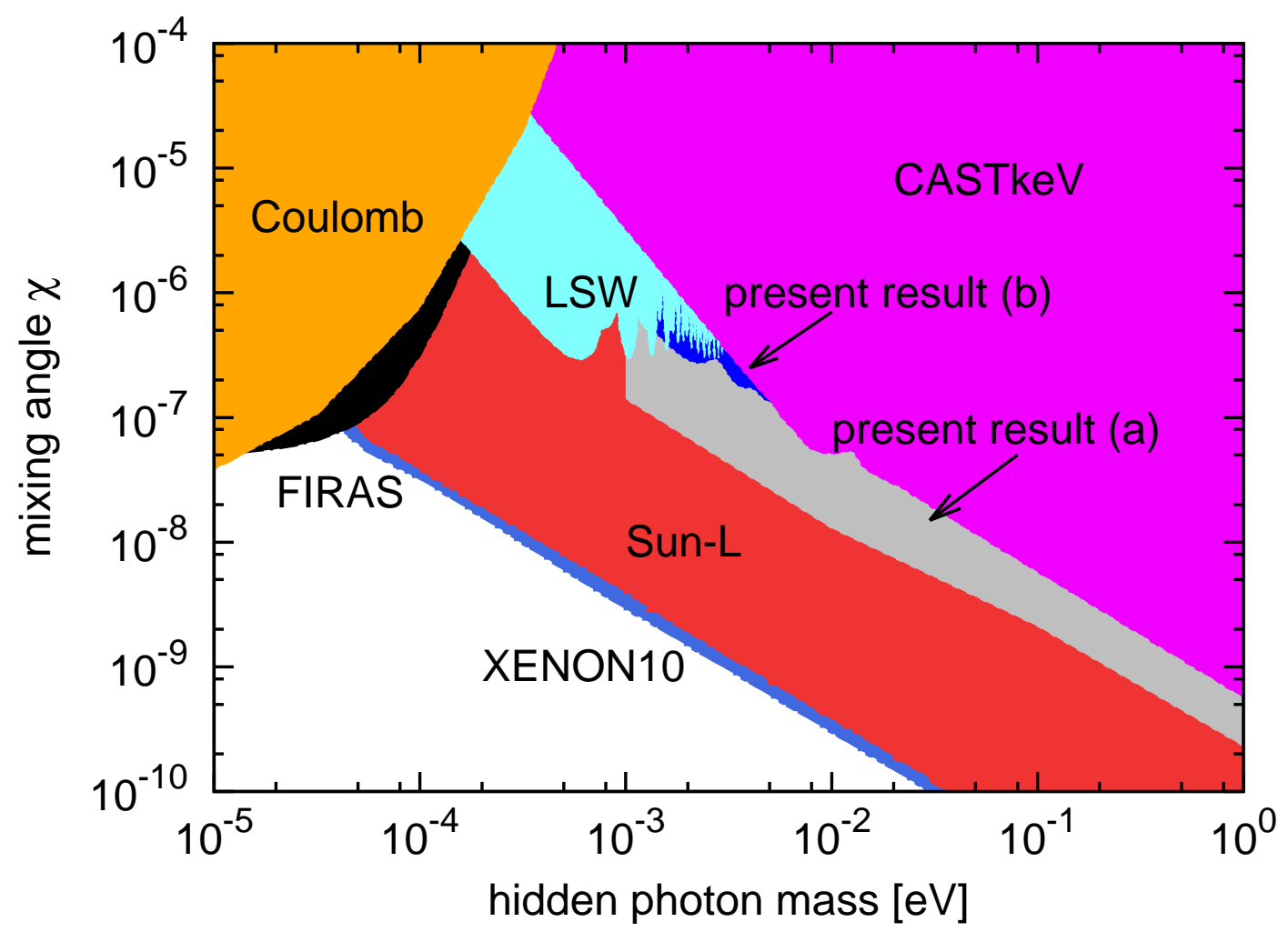

Figure 5. $95 \%$ Confidence level upper limits to the mixing angle $\chi$ set by this experiment. Present result (a) is obtained on the basis of the newer refined solar hidden photon flux calculation[21,22] and (b) on the basis of the older conservative estimations[16], equations(1.6) and (1.7). Filled areas are excluded by other experiments. The regions excluded by precision measurements of Coulomb's law[5, 6], LSW(Light Shining through Walls) experiments[9-12], the CAST experiment[16] and FIRAS CMB spectrum[20] are marked "Coulomb", "LSW", "CAST keV" and "FIRAS", respectively. The solar luminosity constraints[30, 32] in the longitudinal channel and XENON10 limits[31] on the longitudinal solar hidden photons are marked "Sun-L" and "XENON10", respectively.

through Walls) experiments[9-12] and the CAST experiment[16] are marked "Coulomb", "LSW" and "CAST keV", respectively. The excluded region by FIRAS CMB spectrum[20] is marked "FIRAS".

After submitting the original manuscript, the authors have learned of the works[30, 32] reporting the calculations of hidden photon emission from the sun and other stars. They emphasized the effect of longitudinal-mode hidden photon emission underestimated in the previous calculations[16, 21, 22], and gave the solar luminosity constraints on the hidden photon parameters, which we added in fig. 5. Another work[31] reanalyzed the published data of XENON10 dark matter search experiment and compared them with the predominant longitudinal-mode solar hidden photon flux to obtain the constraints on the hidden photon parameters. The constraints is also added in fig. 5 .

Even with these new works, on the other hand, the present result stays unchanged 
because it solely rely on the transverse-mode solar hidden photon emission flux[16, 21, $22]$, which is valid as far as the transverse-mode is concerned[30, 33].

\section{Conclusion}

We have searched for solar hidden photons in the eV energy range using a dedicated detector for the first time. The detector was attached to the Tokyo axion helioscope, Sumico which has a mechanism to track the sun. From the result of the measurement, there is no evidence of the existence of hidden photons and we set a limit on photonhidden photon mixing parameter $\chi$ depending on the hidden photon mass $m_{\gamma^{\prime}}$. The present result improved the existing limits given by the LSW experiments and the CAST experiment in the hidden photon mass region between $10^{-3}$ and $1 \mathrm{eV}$. With recent new calculations of the longitudinal-mode hidden photon, more stringent limits came out by the solar luminosity consideration and also by the reanalysis of XENON10 data, while the present result is based on the search for the transverse-mode solar hidden photons.

\section{Acknowledgements}

The authors thank all the historical members of Sumico experiment because the present hidden photon search solely owes them for her altazimuth tracking system. T. Horie acknowledges support by Advanced Leading Graduate Course for Photon Science (ALPS) at the University of Tokyo. T. Mizumoto would like to thank support by the Grantin-Aid for JSPS Fellows Grant Number 229033 and by the Global COE Program "the Physical Sciences Frontier", MEXT, Japan. R. Ohta would like to thank support by the Grant-in-Aid for JSPS Fellows Grant Number 207600. This reaserch is supported by the Grant-in-Aid for challenging Exploratory Research by MEXT, Japan, and by the Research Center for the Early Universe, School of Science, the University of Tokyo. The authors acknowledge useful communication with J. Redondo on the solar hidden photon flux.

\section{References}

[1] M. Goodsell and A. Ringwald, Light hidden-sector U(1)s in string compactifications, 2010 Fortsch. Phys. 58716.

[2] L. Okun, Limits on electrodynamics: paraphotons?, 1982 Sov. Phys. JETP 56502.

[3] B. Holdom, Two U(1)'s and $\epsilon$ charge shifts, 1986 Phys. Lett. B 166196.

[4] R. Foot, X. He, Comment on Z - Z' mixing in extended gauge theories, 1991 Phys. Lett. B 267509.

[5] E. Williams, J. Faller, H. A. Hill, New Experimental Test of Coulomb's Law: A Laboratory Upper Limit on the Photon Rest Mass, 1971 Phys. Rev. Lett. 26721.

[6] D. F. Bartlett and S. Loegl, Limits on an Electromagnetic Fifth Force, 1988 Phys. Rev. Lett. 612285. 
[7] V. Popov, O. Vasil'ev, Deviations from Electrodynamics: Sun and Laser, 1991 Europhys. Lett. 157.

[8] V. Popov, On the experimental search for photon mixing, 1999 Turkish Journal of Physics 23943.

[9] K. Ehret et al. (ALPS Collaboration), New ALPS results on hidden-sector lightweights, 2010 Phys. Lett. B 689 149, arXiv. 1004 .1313v1 [hep-ex].

[10] M. Fouche et al. (BMV Collaboration), Search for photon oscillations into massive particles, 2008 Phys. Rev. D 78 032013, arXiv:0808.2800v1 [hep-ex].

[11] M. Ahlers et al., Laser experiments explore the hidden sector, 2008 Phys. Rev. D 77 095001, arXiv:0711.4991v1 [hep-ph], A. S. Chou et al. (GammeV Collaboration), Search for axion- like particles using a variable baseline photon regeneration technique, 2008 Phys. Rev. Lett. 100 080402, arXiv:0710.3783 [hep-ex].

[12] A. Afanasev et al. (LIPSS Collaboration), New experimental limit on photon hidden-sector paraphoton mixing, 2009 Phys. Lett. B 679 317, arXiv:0810.4189v1 [hep-ex].

[13] R. Horvat, D. Kekez, M. Krcmar, Z. Krecak, A. Ljubicic, Constraining solar hidden photons using HPGe detector, arXiv:1210.1043v2 [hep-ex].

[14] K. Zioutas, et al., First Results from the CERN Axion Solar Telescope, 2005 Phys. Rev. Lett. 94121301.

[15] S. Andriamonje, et al., An improved limit on the axionphoton coupling from the CAST experiment, 2007 JCAP 04010.

[16] J. Redondo, Helioscope bounds on hidden sector photons, 2008 JCAP 07 008, arXiv:0801.1527 [hep-ph], S. N. Gninenko and J. Redondo, On search for eV hidden-sector photons in Super-Kamiokande and CAST experiments, 2008 Phys. Lett. B 664 180, arXiv:0804.3736v1 [hep-ex].

[17] S. Davidson, M. Peskin, Astrophysical bounds on millicharged particles in models with a paraphoton, 1994 Phys. Rev. D 492114.

[18] S. Davidson, S. Hannestad, G. Raffelt, Updated bounds on milli-charged particles, 2000 JHEP 0005003.

[19] S. Dubovsky, D. Gorbunov, G. Rubtsov, Narrowing the window for millicharged particles by CMB anisotropy, 2004 JETP Lett. 791.

[20] A. Mirizzi, J. Redondo and G. Sigl, Microwave background constraints on mixing of photons with hidden photons, 2009 JCAP 03 026, arXiv:0901.0014[hep-ph].

[21] D. Cadamuro and J. Redondo, Hidden Photons from the Sun, arXiv:1010.4689v1 [hep-ph].

[22] J. Redondo, The Sun in Hidden Photons, arXiv:1202.4932v1 [hep-ph].

[23] R. Peccei, H. Quinn, CP Conservation in the Presence of Pseudoparticles, 1977 Phys. Rev. Lett. 381440.

[24] R. Peccei, H. Quinn, Constraints imposed by CP conservation in the presence of pseudoparticles, 1977 Phys. Rev. D 161791. 
[25] R. Ohta, et al., The Tokyo Axion Helioscope, 2012 Nucl. Instr. Meth. A 67073.

[26] S. Moriyama, et al., Direct search for solar axions by using strong magnetic field and X-ray detectors, 1998 Phys. Lett. B 434147.

[27] Y. Inoue, et al., Search for sub-electronvolt solar axions using coherent conversion of axions into photons in magnetic field and gas helium, 2002 Phys. Lett. B 53618.

[28] Y. Inoue, et al., Search for solar axions with mass around $1 \mathrm{eV}$ using coherent conversion of axions into photons, 2008 Phys. Lett. B 66893.

[29] http://aa.usno.navy.mil/software/novas/novas_info.php

[30] H. An, M. Pospelov, J. Pradler, New stellar constraints on dark photons, arXiv:1302.3884 [hep-ph].

[31] H. An, M. Pospelov, J. Pradler, Dark Matter Detectors as Dark Photon Helioscopes, arXiv:1304.3461 [hep-ph].

[32] J. Redondo, G. Raffelt, Solar constraints on hidden photons re-visited, arXiv:1305.2920 [hep-ph].

[33] J. Redondo, private communication. 